\title{
Estudo de Aplicativos Móveis para Deficientes Visuais no Âmbito Acadêmico
}

\author{
Janaina Cristina da Silva, Rafael Jeferson Pezzuto Damaceno, Juliana Cristina \\ Braga \\ Universidade Federal do ABC (UFABC) - Santo André - SP - Brasil \\ Centro de Matemática, Computação e Cognição - CMCC \\ Grupo de Pesquisa INTERA \\ \{janaina.cristina, rafael.damaceno, juliana.cristina\}@ufabc.edu.br
}

\begin{abstract}
The present work aims to study about mobile application in the academic environment by visually impaired, due to the fact that the mobile devices shows great potential of use by these users to improve the school life. However, these applications have low accessibility, making its use hard for the visually impaired, due to the fact that the development of these applications their specific needs and abilities were not taken into account. Come from the problems identified in this study, it will be possible to trace strategies and solutions for these applications to be used in support of affordable education.
\end{abstract}

Resumo. $O$ presente trabalho tem como objetivo o estudo de aplicativos móveis a serem utilizados no âmbito acadêmico pelos deficientes visuais, devido ao fato dos dispositivos móveis apresentarem grande potencial de uso por parte destes usuários para a melhoria da vida escolar. No entanto, os aplicativos existentes possuem baixa acessibilidade, dificultando assim o seu uso pelos deficientes visuais, pois no desenvolvimento destes aplicativos as suas necessidades e capacidades específicas não foram levados em conta. A partir dos problemas identificados no estudo, será possível traçar estratégias e soluções para que esses aplicativos sejam utilizados em prol da educação acessivel.

\section{Introdução}

Segundo dados do Censo demográfico de 2010, divulgados pelo Instituto Brasileiro de Geografia e Estatística (IBGE), em abril de 2012, mais de 45,6 milhões de brasileiros declararam ter alguma deficiência. Deste total, 35,7 milhões de pessoas possuíam deficiência visual, e mais de 506 mil se declararam cegas. Sendo esta deficiência visual na sua mais variada intensidade, desde a uma deficiência leve à perda total da visão, em virtudes de causas congênitas ou hereditárias, tida como uma situação irreversível.

Para estes deficientes, a tecnologia assistiva tende a ser usada como instrumento de acessibilidade e inclusão, proporcionando-os alguns benefícios como: maior independência e qualidade de vida.

No que se refere à tecnologia assistiva, no Brasil, foi proposto o seguinte conceito: "Tecnologia Assistiva é uma área do conhecimento, de característica interdisciplinar, que engloba produtos, recursos, metodologias, estratégias, práticas e serviços que objetivam promover a funcionalidade, relacionada à atividade $\mathrm{e}$ 
participação de pessoas com deficiência, incapacidades ou mobilidade reduzida, visando sua autonomia, independência, qualidade de vida e inclusão social" (Brasil, 2007).

$\mathrm{Na}$ tecnologia assistiva, os dispositivos móveis, como por exemplo, os smartphones e tablets, por serem ubíquos e terem hardwares avançados, podem ser importantes aliados nos benefícios citados anteriormente (Braga, et al., 2012). Estudos têm investigado aplicações que estes aparelhos podem ter na vida de deficientes visuais, bem como: locomoção em ambientes internos e externos, leitura de documentos de texto entre outras.

No âmbito acadêmico, os dispositivos móveis podem ser muito úteis para auxiliar o deficiente visual no aprendizado e contribuir o para o ensino acessível. Podese citar como exemplo desses benefícios: gravação de aulas, envio de e-mails com dúvidas, acesso aos AVAs (Ambiente Virtual de Aprendizagem), tradução de artigos, leitores de telas para transformar as aulas de texto para voz, dentre outros. Destaca-se que a grande vantagem do uso destes dispositivos é que esses benefícios podem ser usufruídos com o uso de somente um único aparelho e de fácil mobilidade.

Dado o exposto, percebeu-se que uma análise poderá ser realizada para entender a forma com que estas tecnologias veem sendo utilizadas pelos Deficientes Visuais, identificando assim os problemas de acessibilidade, e propondo futuras soluções.

Este trabalho encontra-se organizado da seguinte forma: Na seção 2, é abordado o objetivo deste estudo. A seção 3 apresenta o conceito sobre acessibilidade para dispositivos móveis e a importância do método de revisão sistemática. Na seção 4, é tratado sobre o método utilizado na pesquisa. Na seção 5 são divulgados os resultados obtidos. A seção 6 expõe a conclusão sobre o presente trabalho. Por fim, são apresentadas as referências de todos os artigos utilizados no presente trabalho.

\section{Objetivo}

O objetivo do presente estudo é a identificação de aplicativos móveis para deficientes visuais no âmbito educacional, assim como analisar o uso pedagógico destes aplicativos e a maneira de como foram utilizados na educação.

\section{Referencial Teórico}

Esta seção procura esclarecer os conceitos que nortearam a realização deste trabalho. Inicialmente é abordada a "acessibilidade de dispositivos móveis", onde é traçado um perfil dos problemas e soluções ao longo do tempo até os dias atuais. Em seguida é explicada a importância do método de revisão sistemática utilizada na pesquisa.

\subsection{Acessibilidade de dispositivos móveis}

O termo "acessibilidade de dispositivos móveis" se refere à capacidade de interagir adequadamente com o sistema operacional (Android, iOS, Windows Phone, entre outros) dos aparelhos. Para garantir que pessoas com deficiência visual interajam adequadamente com os dispositivos móveis, há uma série de ferramentas de acessibilidade disponíveis nos sistemas operacionais destes aparelhos, tais como leitor de tela, lente de aumento e controle de contraste (Leporini et al., 2012). O leitor de tela apresenta em voz as informações do sistema que estão em foco. A lente de aumento amplia os elementos gráficos da tela e o alto contraste inverte as suas cores. 
No entanto, mesmo à época dos dispositivos mais antigos, que não possuíam tela sensível ao toque, já havia problemas de acessibilidade, ou seja, falhas nas ferramentas ou mesmo sua ausência, que impedia o usuário de executar de forma satisfatória as funções do aparelho. Problemas como teclado físico pequeno com muitas teclas ou que possuíam poucas teclas para várias funções (Guerreiro et al., 2009) e a ausência de leitor de tela em alguns dispositivos (Kane et al., 2009) são alguns problemas abordados na literatura.

Ao longo do tempo, as falhas destas ferramentas foram corrigidas e formas alternativas de acesso e entrada de informações foram desenvolvidas. No caso da entrada de texto, como solução, pode-se mencionar o exemplo do NavTap, método que permite digitar caracteres de texto usando o controle direcional do dispositivo, possibilitando ao usuário navegar por submenus de caracteres de forma bastante intuitiva (Guerreiro et al., 2009). No caso de sistemas operacionais Symbian, foram implementadas alternativas de acesso por meio de menus com recepção de comandos de voz e com retorno ao usuário por meio de leitor de tela.

Com o advento das telas sensíveis ao toque, outras questões de acessibilidade vieram à tona, dado o maior apelo visual para interagir com esta tecnologia. $\mathrm{Na}$ interação com leitor de tela, por exemplo, o usuário executa gestos de toque, que são um conjunto de movimentos realizados com os dedos, na tela, para desencadear ações do sistema operacional. Estudos têm verificado falha ou dificuldade para aprender a realizar estes gestos, resultando em ações inesperadas do sistema (Kane, S.K. et al., 2011; Leporini et al., 2012).

Atualmente é possível para pessoas com deficiência visual usar dispositivos móveis mais recentes por meio das ferramentas de acessibilidade neles disponíveis. No entanto, a baixa apropriação das tecnologias atuais por parte de pessoas com deficiência visual e as características próprias na forma de realização dos gestos (Kane, S.K. et al. 2011) tem dificultado o uso dos dispositivos móveis.

\subsection{Revisão Sistemática}

A metodologia utilizada nas revisões sistemáticas teve início na medicina com Archie Cochrane (1909-1988) médico e pesquisador britânico, sendo agora utilizada por diversas outras áreas do conhecimento. $\mathrm{Na}$ área da Computação (Engenharia de Software) a revisão sistemática foi introduzida por Barbara Kitchenham em 2004. Para Kitchenham, a revisão sistemática da literatura é um meio de identificar, avaliar e interpretar todas as pesquisas disponíveis relevantes para uma determinada questão ou área de tópico, ou fenômeno de interesse.

A revisão sistemática possui 3 (três) fases: Planejamento, Condução e Relatório. Segundo Kitchenham (2004), na fase de Planejamento o pesquisador faz uma identificação das necessidades de uma revisão sistemática e um protocolo de revisão é desenvolvido. Neste protocolo são determinados no início da pesquisa os critérios de inclusão e exclusão para a seleção dos artigos. Na fase de Condução, é feita uma identificação da pesquisa, seleção dos estudos e avaliação da qualidade destes estudos, em seguida os dados relevantes encontrados nos trabalhos selecionados são extraídos e depois sintetizados. A última fase é a fase Relatório onde se escreve os resultados de todas as fases anteriores. 


\section{Métodos}

Para atingir o objetivo desse trabalho que foi identificar aplicativos móveis para deficientes visuais no âmbito acadêmico, utilizou-se como metodologia, uma revisão sistemática de literatura. Para tal fim, foi utilizada a metodologia de Revisão Sistemática (método proposto por Kitchenham, 2004), que levantou artigos publicados com o propósito de responder as questões desta pesquisa: "Existem aplicativos móveis no âmbito educacional para deficiente visual? Se sim, como eles foram utilizados?".

Entre as bases de dados disponíveis, foram selecionadas as principais bases de dados de pesquisa internacional da área. As selecionadas foram: ACM Digital Library, IEEEXplore Digital Library, ISI Web of Science, Elsevier (Science Direct), Springer e Google Scholar.

Os idiomas escolhidos foram: o inglês e o português. O inglês por ser amplamente utilizado no meio acadêmico e o português por ser língua nativa, tem- se a intenção de se encontrar trabalhos brasileiros sobre o tema deste trabalho nas bases de dados escolhidas, uma vez que o principal congresso da área de IHC possui indexação em uma das bases escolhidas.

Quanto às palavras-chave foram utilizadas as seguintes palavras: Accessibility; Blind; "Mobile Device" e Education. Essas palavras-chaves foram buscadas juntamente com seus termos e sinônimos relacionados (ver Tabela 1), servindo de parâmetros para as buscas de trabalhos, de maneira a responder as questões de pesquisa.

Tabela 1. Definição dos termos e sinônimos relacionados

\begin{tabular}{|c|c|c|c|}
\hline Accessibility & Blind & "Mobile device" & Education \\
\hline $\begin{array}{l}\text { Accessible } \\
\text { Technology } \\
\text { Assistive }\end{array}$ & $\begin{array}{l}\text { "Visually Impaired" } \\
\text { "Visually disabled" }\end{array}$ & $\begin{array}{l}\text { Android } \\
\text { IOS } \\
\text { Ipad } \\
\text { Iphone } \\
\text { "Mobile Phone" } \\
\text { Smartphone } \\
\text { Tablet } \\
\text { Touchscreen } \\
\text { Ubiquity }\end{array}$ & $\begin{array}{l}\text { Acquirements } \\
\text { Training } \\
\text { Breeding } \\
\text { Accomplishment } \\
\text { Teach } \\
\text { Learning } \\
\text { Guideline } \\
\text { Pedagogical } \\
\text { Classroom }\end{array}$ \\
\hline
\end{tabular}

Na definição das Strings de Busca (ou Expressão de Busca), os operadores lógicos OR e AND foram utilizados, de modo que a combinação das palavras-chaves com os operadores lógicos, indicaram ao sistema de busca como devem ser feitas as combinações das strings. As strings passaram por testes, sendo construídas em idioma inglês, e foram adaptadas conforme a especificidade de cada base. No entanto, houve algumas dificuldades com relação às strings, de maneira a manter a mesma consistência para todas as bases de dados. Esta dificuldade se deu devido às limitações de caracteres a serem inseridos nos campos de pesquisa dessas bases de dados. Com a submissão das strings nas fontes de busca eletrônica, um levantamento de trabalhos foi realizado, baseado na leitura do título e resumo (e quando necessário, a leitura de outras partes do trabalho, como conclusão e introdução, por exemplo).

Dentre os critérios de inclusão foram adotados trabalhos: com qualquer data de publicação; sobre aplicativos utilizados no âmbito educacional por deficientes visuais; que abordaram sobre acessibilidade de dispositivos móveis, e que tenham utilizados 
sistema operacional Android e/ou iOS. Foram excluídos trabalhos que não tinham relação quanto à utilização de dispositivos móveis por deficientes visuais e trabalhos sobre aplicativos que não foram utilizados no âmbito acadêmico, assim como trabalhos duplicados e cujo idioma não tenha sido o inglês ou português.

Com a pré-seleção dos trabalhos, outras partes dos trabalhos foram lidas e os critérios de exclusão e inclusão foram aplicados, de forma a se fazer uma nova seleção. Como etapa final, todos os trabalhos incluídos foram lidos na íntegra e uma análise destes trabalhos foi feita, o que é descrito na seção seguinte.

\section{Resultados}

Dos 230 trabalhos encontrados, apenas 8 (oito) artigos atenderam aos critérios de inclusão, com isso foi feita uma descrição individual de cada estudo identificando as suas principais características. A tabela 2 sintetiza os resultados dos trabalhos incluídos no presente estudo, identificando: Autores/Ano de Publicação, Tema do Aplicativo, Local de Avaliação, Número de Participantes, Idades e o Tipo de Deficiência dos participantes que utilizaram os aplicativos.

Tabela 2. Características dos participantes com relação a cada estudo

\begin{tabular}{|c|c|c|c|c|c|}
\hline $\begin{array}{c}\text { Autores/ } \\
\text { Ano de } \\
\text { Publicação }\end{array}$ & Tema & Local de Avaliação & $\begin{array}{l}\text { Número de } \\
\text { Participantes }\end{array}$ & Idades & Deficiência \\
\hline $\begin{array}{l}\text { Milne et } \\
\text { al.(2014) }\end{array}$ & $\begin{array}{l}\text { Conjunto de } 4 \text { jogos } \\
\text { (aplicativos para entrada de } \\
\text { texto) }\end{array}$ & $\begin{array}{l}\text { Países: EUA e Panamá. } \\
\text { Vídeo Conferência. }\end{array}$ & $\begin{array}{l}8 \text { conjuntos de } \\
\text { participantes } \\
\text { (pais e filhos) }\end{array}$ & $5-8$ anos & $\begin{array}{l}\text { cego e de } \\
\text { baixa visão }\end{array}$ \\
\hline $\begin{array}{l}\text { Southern et } \\
\text { al.(2012) }\end{array}$ & $\begin{array}{l}\text { Aplicativo para } \\
\text { Entrada de Texto }\end{array}$ & - & 11 & $\begin{array}{l}\text { 28-65 anos } \\
\text { média: } \\
\text { 47,4 anos }\end{array}$ & $\begin{array}{l}9 \text { cegos } \\
\text { nascença }\end{array}$ \\
\hline $\begin{array}{l}\text { Jr and } \\
\text { Hayden } \\
(2010)\end{array}$ & $\begin{array}{l}\text { Protótipo de uma tecnologia } \\
\text { assistiva tomadora de nota em } \\
\text { sala de aula }\end{array}$ & sala de aula & 6 & - & $\begin{array}{l}2 \text { totalmente } \\
\text { cegos }\end{array}$ \\
\hline $\begin{array}{l}\text { Zapirain et } \\
\text { al.(2010) }\end{array}$ & $\begin{array}{l}\text { Algoritmo para leitura de } \\
\text { esquema de circuito } \\
\text { eletrônico }\end{array}$ & - & - & - & $\begin{array}{l}\text { deficientes } \\
\text { visuais e } \\
\text { surdos }\end{array}$ \\
\hline $\begin{array}{l}\text { Jayant et } \\
\text { al. }(2010)\end{array}$ & $\begin{array}{l}\text { Aplicativo para leitura e } \\
\text { escrita de caracteres em } \\
\text { Braile em dispositivo móvel }\end{array}$ & - & 9 & $\begin{array}{l}\text { média: } \\
\text { 47,5 anos }\end{array}$ & $\begin{array}{l}\text { 3- cegos } \\
6 \text {-surdo-cega }\end{array}$ \\
\hline $\begin{array}{l}\text { Issa et al. } \\
(2009)\end{array}$ & $\begin{array}{l}\text { Estudo para a criação } \\
\text { de um aplicativo para } \\
\text { interpretação de imagens }\end{array}$ & - & - & - & $\begin{array}{l}\text { deficientes } \\
\text { visuais }\end{array}$ \\
\hline $\begin{array}{l}\text { Mehigan,J. } \\
\text { T.(2009) }\end{array}$ & $\begin{array}{l}\text { Pesquisa sobre a criação de } \\
\text { um sistema de aprendizado } \\
\text { utilizável e acessível }\end{array}$ & - & - & - & $\begin{array}{l}\text { cegos / } \\
\text { deficientes } \\
\text { visuais }\end{array}$ \\
\hline $\begin{array}{l}\text { Raisamo et } \\
\text { al.(2007) }\end{array}$ & Jogo (aplicativo acessível) & $\begin{array}{l}\text { Sala de aula vazia, } \\
\text { onde a configuração de } \\
\text { teste foi construída. }\end{array}$ & 7 & $\begin{array}{c}12 \text { a } 13 \\
\text { anos }\end{array}$ & $\begin{array}{l}3 \text { totalmente } \\
\text { cegos; } \\
4 \text { amblíopes. }\end{array}$ \\
\hline
\end{tabular}

Com a revisão sistemática, foi possível encontrar alguns estudos que atenderam aos objetivos deste trabalho. A concentração maior destes estudos foi nos Estados Unidos da América, seguida pelos países europeus. Pode-se observar uma semelhança 
entre os temas dos estudos incluídos, pois os mais abordados foram: entrada de textos e jogos educativos em Braille. Entre os estudos que informaram os números de participantes, a média foi de 8 (oito) participantes por estudo. O público-alvo dos aplicativos variou de crianças a idosos, sendo a maioria deles voltados para crianças, e quanto mais velha a pessoa, mais complexos eram os aplicativos. Apesar da expressão 'deficiência visual' se referir ao espectro que vai da cegueira até a visão subnormal (ou, baixa visão), em quase todos os trabalhos este espectro não foi informado, dando a entender que muitos participantes se utilizavam de recursos visuais para ler como: lente de aumento e percepção luminosa.

A tabela 3 sintetiza o uso pedagógico dos aplicativos dos trabalhos incluídos e como esses aplicativos foram utilizados na educação.

Tabela 3. Síntese dos aplicativos na educação

(continua)

\begin{tabular}{|c|c|c|c|c|}
\hline $\begin{array}{l}\text { Autores/ } \\
\text { Aplicativo }\end{array}$ & $\begin{array}{c}\text { Objetivo } \\
\text { Pedagógico }\end{array}$ & Dificuldades Relatadas & Facilidades & $\begin{array}{c}\text { Benefícios para a } \\
\text { educação }\end{array}$ \\
\hline $\begin{array}{l}\text { Milne et } \\
\text { al.(2014) / } \\
\text { Braille Play }\end{array}$ & $\begin{array}{l}\text { Promover a } \\
\text { alfabetização de } \\
\text { crianças com } \\
\text { diferentes graus de } \\
\text { deficiência visual } \\
\text { em Braille }\end{array}$ & $\begin{array}{l}\text { Gestos multi-touch podem não ser } \\
\text { adequados para crianças devido à } \\
\text { dificuldade em batida de dois dedos } \\
\text { na tela. } \\
\text { Crianças apresentam uma } \\
\text { diminuição do interesse no jogo } \\
\text { após } 2 \text { semanas de interação }\end{array}$ & $\begin{array}{l}\text { As crianças foram } \\
\text { capazes de interagir } \\
\text { de forma autônoma } \\
\text { com o aplicativo }\end{array}$ & $\begin{array}{l}\text { O aplicativo } \\
\text { promove a melhoria } \\
\text { da capacidade de ler } \\
\text { e escrever letras em } \\
\text { Braille. }\end{array}$ \\
\hline $\begin{array}{l}\text { Southern et } \\
\text { al.(2012) / } \\
\text { BrailleTouch }\end{array}$ & $\begin{array}{l}\text { Promover o } \\
\text { aprendizado do } \\
\text { alfabeto Braille } \\
\text { através da } \\
\text { digitação em } \\
\text { dispositivos } \\
\text { móveis }\end{array}$ & $\begin{array}{l}\text { Em cada dispositivo móvel } \\
\text { utilizado nos testes, o mesmo gesto } \\
\text { foi interpretado de maneira } \\
\text { diferente. } \\
\text { Os tamanhos das telas dos } \\
\text { dispositivos influenciaram no } \\
\text { desempenho dos usuários, assim } \\
\text { como as mãos dos usuários } \\
\text { tenderam a se mover lentamente } \\
\text { pela tela touchscreen devido à falta } \\
\text { de um referencial tátil nas telas. E a } \\
\text { ausência de retorno de voz para a } \\
\text { confirmação de inserção de } \\
\text { caractere. }\end{array}$ & $\begin{array}{l}\text { Vantajosa velocidade } \\
\text { significativa sobre } \\
\text { outros teclados } \\
\text { touchscreen braille } \\
\text { (Multi-tap, NavTap e } \\
\text { BrailleTap). Substitui } \\
\text { o uso de tecnologias } \\
\text { assistivas caras com } \\
\text { o uso do aplicativo } \\
\text { nos dispositivos } \\
\text { móveis do usuário. }\end{array}$ & $\begin{array}{l}\text { Mesmo sem } \\
\text { conhecimento } \\
\text { prévio em Brailler, é } \\
\text { possível aprender o } \\
\text { alfabeto em algumas } \\
\text { horas e ganhar } \\
\text { velocidade na } \\
\text { digitação. }\end{array}$ \\
\hline $\begin{array}{l}\mathrm{Jr} \text { and Hayden } \\
(2010) / \\
\text { Protótipo (The } \\
\text { Note-Taker) }\end{array}$ & $\begin{array}{l}\text { Aumentar as } \\
\text { capacidades de } \\
\text { aprendizagem e de } \\
\text { anotações de } \\
\text { alunos cegos em } \\
\text { sala de aula }\end{array}$ & $\begin{array}{l}\text { Condição de luminosidade } \\
\text { desfavorável na sala de aula, falta } \\
\text { de limpeza do quadro-negro / } \\
\text { quadro-branco, resultando em uma } \\
\text { representação menos do que } \\
\text { desejável e problemas significativos } \\
\text { com o uso de alguns ampliadores de } \\
\text { tela para tomar manuscritas notas } \\
\text { sobre Tablet. }\end{array}$ & $\begin{array}{l}\text { Ganho de tempo } \\
\text { devido a não } \\
\text { necessidade de: } \\
\text { configuração prévia } \\
\text { do equipamento em } \\
\text { sala de aula e; } \\
\text { revisão das gravações } \\
\text { ou anotações fora da } \\
\text { sala de aula. } \\
\end{array}$ & $\begin{array}{l}\text { Os alunos vão poder } \\
\text { acompanhar as aulas } \\
\text { independentemente } \\
\text { do ritmo de aula do } \\
\text { professor }\end{array}$ \\
\hline $\begin{array}{l}\text { Zapirain et } \\
\text { al.(2010) / } \\
\text { - }\end{array}$ & $\begin{array}{l}\text { Fornecer uma } \\
\text { descrição textual } \\
\text { das sequências dos } \\
\text { componentes } \\
\text { eletrônicos e da } \\
\text { posição no } \\
\text { esquema geral }\end{array}$ & - & $\begin{array}{l}\text { O grau de dificuldade } \\
\text { em relação ao tempo } \\
\text { necessário para } \\
\text { dominar o aplicativo } \\
\text { é baixo }\end{array}$ & $\begin{array}{l}\text { Promover o acesso } \\
\text { de deficientes } \\
\text { visuais a cursos de } \\
\text { nível técnico, onde a } \\
\text { utilização de } \\
\text { esquemas de } \\
\text { circuitos eletrônicos } \\
\text { são comuns }\end{array}$ \\
\hline
\end{tabular}


CBIE-LACLO 2015

Anais do XXVI Simpósio Brasileiro de Informática na Educação (SBIE 2015)

Tabela 3. Síntese dos aplicativos na educação

(conclusão)

\begin{tabular}{|c|c|c|c|c|}
\hline $\begin{array}{l}\text { Autores/ } \\
\text { Aplicativo }\end{array}$ & Objetivo Pedagógico & Dificuldades Relatadas & Facilidades & $\begin{array}{l}\text { Benefícios para a } \\
\text { educação }\end{array}$ \\
\hline $\begin{array}{l}\text { Jayant et } \\
\text { al.(2010) / } \\
\text { V-Braille }\end{array}$ & $\begin{array}{l}\text { Ajudar as crianças e } \\
\text { seus pais a aprender a } \\
\text { ler e escrever letras em } \\
\text { Braille. }\end{array}$ & $\begin{array}{l}\text { Para diferenciar entre as } \\
\text { seis áreas de ponto em uma } \\
\text { única célula Braille, o uso } \\
\text { de uma divisória tátil no } \\
\text { telefone foi sugerido. }\end{array}$ & $\begin{array}{l}\text { Não utiliza nenhum } \\
\text { hardware ou } \\
\text { dispositivo adicional. }\end{array}$ & $\begin{array}{l}\text { Permite a leitura de } \\
\text { caracteres individuais } \\
\text { e sentenças com o uso } \\
\text { do aplicativo em } \\
\text { dispositivo móvel }\end{array}$ \\
\hline $\begin{array}{l}\text { Issa et al. } \\
(2009) / \\
-\end{array}$ & $\begin{array}{l}\text { Proporcionar uma } \\
\text { apresentação acessível } \\
\text { de informações visuais } \\
\text { com o uso de um } \\
\text { feedback táctil. }\end{array}$ & - & - & $\begin{array}{l}\text { Tornar informações } \\
\text { visuais acessíveis, de } \\
\text { forma a manter o } \\
\text { aspecto bidimensional } \\
\text { de um documento e } \\
\text { desenhá-la na mente } \\
\text { dos deficientes visuais. }\end{array}$ \\
\hline $\begin{array}{l}\text { Mehigan,J. } \\
\text { T.(2009) / } \\
\text { - }\end{array}$ & $\begin{array}{l}\text { Criação de um sistema } \\
\text { de aprendizagem móvel } \\
\text { utilizável e acessível, } \\
\text { utilizando dispositivos } \\
\text { móveis touchscreen e } \\
\text { acelerômetro. }\end{array}$ & - & $\begin{array}{l}\text { Interação com o jogo } \\
\text { através de movimentos } \\
\text { específicos com o } \\
\text { dispositivo móvel, } \\
\text { retornos tátil, vibratório } \\
\text { e de áudio seria } \\
\text { utilizados e o uso de } \\
\text { telas touchscreen } \\
\text { eliminaria a utilização } \\
\text { de botões } \\
\text { complementares. }\end{array}$ & $\begin{array}{l}\text { Aproximar os alunos } \\
\text { deficientes visuais dos } \\
\text { não deficientes, em } \\
\text { atividades comuns } \\
\text { dentro e fora da sala de } \\
\text { aula através do uso de } \\
\text { dispositivo móvel. }\end{array}$ \\
\hline $\begin{array}{l}\text { Raisamo et } \\
\text { al.(2007)/ } \\
\text { - }\end{array}$ & $\begin{array}{l}\text { Ensinar habilidades } \\
\text { (motoras, táteis e } \\
\text { direcionais) para as } \\
\text { crianças de uma forma } \\
\text { simples e sob a forma } \\
\text { de um jogo de memória } \\
\text { clássico. }\end{array}$ & $\begin{array}{l}\text { Os testes com relação à } \\
\text { usabilidade e jogabilidade } \\
\text { do jogo foram realizados } \\
\text { em adolescentes, e não em } \\
\text { criança, com diferentes } \\
\text { graus de deficiência visual } \\
\text { e idades de } 12-13 \text { anos, } \\
\text { pois os testes poderiam } \\
\text { sobrecarregar as crianças e } \\
\text { um feedback as perguntas e } \\
\text { dificuldades seriam } \\
\text { melhores interpretadas por } \\
\text { adolescentes. }\end{array}$ & $\begin{array}{l}\text { O artigo provou que o } \\
\text { gamepad é um } \\
\text { dispositivo utilizável } \\
\text { por crianças de } 12-13 \\
\text { anos, de fácil } \\
\text { disponibilidade e de } \\
\text { compra e sua qualidade } \\
\text { de feedback tátil é } \\
\text { suficiente para os jogos } \\
\text { do estudo. }\end{array}$ & $\begin{array}{l}\text { Os benefícios de jogos } \\
\text { multimodais para } \\
\text { crianças cegas são que } \\
\text { o jogo pode motivar a } \\
\text { usar os computadores } \\
\text { também para outros } \\
\text { fins e, assim, apoiar o } \\
\text { desenvolvimento das } \\
\text { habilidades } \\
\text { computacionais. }\end{array}$ \\
\hline
\end{tabular}

A seguir são apresentadas algumas informações complementares aos estudos relacionados nas tabelas 2 e 3 :

O estudo de Milne et al. (2014) consistiu no desenvolvimento do aplicativo BraillePlay. Nas fases de testes foram utilizados cinco telefones de plataforma Android com três jogos: VBReader, VBWriter e VBHangman. Tanto pai como filhos utilizavam os mesmos aplicativos, mas ambos com rotulações diferentes, versão criança e versão pai. Para as avaliações foram utilizados videoconferência devido à distância entre autores e participantes, e uma análise do tempo de entrada e precisão de cada jogo com exceção do Hangman foram feitas.

O estudo de Southern et al.(2012) abordou o desenvolvimento do aplicativo BrailleTouch a ser usado em dispositivos móveis por deficientes visuais. Como método de avaliação foi testado as velocidades de escrita do mesmo aplicativo, em 3 dispositivos móveis diferentes: tablet, smartphone e PACmate, num total de cinco sessões. Com sessões de aproximadamente 90 minutos, sendo que cada dispositivo era 
testado por aproximadamente 20 minutos. O período de pausa entre as sessões eram de 90 minutos e não mais que 3 dias. As sentenças a serem digitadas pelos participantes eram passadas através de áudio, com o uso de um sintetizador de voz.

O estudo de Jr and Hayden (2010) visou o desenvolvimento de um anotador portátil (The Note-Taker), uma tecnologia de apoio que permite aos alunos que são legalmente cegos tomar notas em sala de aula, sendo este podendo ser facilmente transportado em sala de aula pelo aluno. O software de aplicação do protótipo foi implementado em linguagem $\mathrm{C}++$ no sistema operacional Windows XP. Este aplicativo emprega o software Microsoft OneNote, para permitir o uso da "tinta digital", ativação da funcionalidade de escrita a mão ou anotações datilografadas.

O estudo de Zapirain et al.(2010) teve como finalidade o desenvolvimento de um algoritmo de código aberto, integrados em uma ferramenta compatível com o Open Office. Este algoritmo aplica técnicas de processamento de imagem digital e técnicas de visão computacional para qualquer esquema de circuito incluído no documento.

O estudo de Jayant et al.(2010) consistiu em um aplicativo que representa caracteres Braille tátil em um dispositivo móvel padrão, usando uma tela plana sensível ao toque e vibração. O aplicativo faz uso do toque e da vibração (de diferentes intensidades, para indicar um ponto em relevo na tela) como forma de interação com o dispositivo. A interface do aplicativo é dividida em 6 partes para reproduzir os seis pontos de uma única célula Braille. Em cada ponto da tela tocado que representa um relevo, o usuário tem um retorno vibratório de diferentes intensidades.

O estudo de Issa et al. (2009) apresentou um trabalho no campo da acessibilidade à informação visual em documentos digitais para deficientes visuais. Eles propuseram como trabalho futuro um processo de impressão termográfica, relatando o modelo final de representação da imagem tátil e com isso a realização de uma avaliação deste conceito, que poderia abrir um novo escopo para acessibilidade multimodal.

O estudo de Raisamo et al. (2006) focou na criação de jogos utilizáveis para crianças com deficiência visual, que fazem uso de dispositivos de vibro-tátil de baixo custo em aplicações multimodais. O jogo foi concebido para ser jogado com um gamepad tátil, onde diferentes vibrações eram para ser lembrado, em vez de sons ou imagens em relevo, que são comuns em jogos de memória para crianças cegas.

\section{Conclusão}

O presente trabalho proporcionou identificar subsídios e aplicativos desenvolvidos para a inclusão dos deficientes visuais no âmbito educacional, apesar das lacunas existentes entre os trabalhos. Os resultados obtidos com a revisão sistemática mostraram que os deficientes visuais podem interagir com os dispositivos móveis e melhorar o seu aprendizado e desempenho rapidamente, devido ao fato de que as suas necessidades foram consideradas. Conclui-se que as duas perguntas de pesquisas deste trabalho foram respondidas conforme os dados apresentados na seção de Resultados.

Os aplicativos em forma de jogos desenvolvem as habilidades motoras, táteis, direcionais, memória, concentração e o raciocínio lógico, além de promover a inclusão digital e recreação, importantes na fase pré-escolar das crianças. A utilização de aplicativos para entrada de textos nos dispositivos móveis, voltados para um público mais velho, acabam estimulando assim como os jogos, a desenvolver as habilidades motoras e promover a inclusão digital. Proporciona também a capacidade de ler e 
produzir textos, e devido aos estímulos auditivos através do sintetizador de voz dos leitores de tela, a audição também é desenvolvida.

Além dos aplicativos apresentados nos trabalhos incluídos, pode-se também citar a existência de alguns aplicativos, não acessíveis, tais como tomador de nota, gravador de voz e calculadoras, com potencial de uso no âmbito acadêmico, disponíveis gratuitamente para serem instalados em dispositivos móveis de sistema operacional Android ou IOS, através do aplicativo App Store existente nestes dispositivos. Seguem alguns destes aplicativos: Gravador \& Gravação de Reunião Free (Gravador Free), Gravador de Voz, Dragon Dictation, Calculadora! e Calculadora +. Contudo o uso destes aplicativos necessitaria a participação de uma segunda pessoa, com a finalidade de instruir o deficiente visual com relação às funcionalidades e layout do aplicativo.

Neste estudo observa-se também que nos trabalhos incluídos, os aplicativos acessíveis em forma de jogos educativos foram voltados para o público infantil, esta geração de jogadores na adolescência assim como os adolescentes atuais, enfrentarão ou já enfrentam desafios em relação à baixa quantidade de aplicativos educativos disponíveis em forma de jogos, destinados a esta geração.

Infelizmente, no Brasil a questão da acessibilidade ganhou impulso somente nas duas últimas décadas através das leis e decretos, e também através das empresas que passaram a ser adaptar a um novo perfil de usuários (Deficientes Visuais), diferentemente dos Estados Unidos onde as primeiras discussões sobre o tema foi em meados de 1973, motivados pelos soldados provenientes de guerras, que passaram a necessitar-se de uma re-inclusão social devido as seus ferimentos. Fatos estes que acabaram refletindo-se na origem dos estudos encontrados.

Portanto, conclui-se que o uso de aplicativos em dispositivos móveis na educação pelos deficientes visuais, contribuem para a sua reabilitação e a sua inclusão escolar. No entanto, ainda existem dificuldades e desafios a serem vencidos nessa área e pesquisas ainda devem ser realizadas para que o uso desses aplicativos na educação seja efetivo.

\section{Referência}

Brasil. Ata VII - Comitê de Ajudas Técnicas - CAT. Secretaria Especial dos Direitos Humanos da Presidência da República (CORDE/SEDH/PR). 2007.

Braga, J. C.; Campi Junior, A.; Damaceno, R. J. P.; Albernaz, N. H. C. Estudo e Relato sobre a Utilização da Tecnologia pelos Deficientes Visuais. In: Simpósio Brasileiro de Fatores Humanos em Sistemas Computacionais 12, 2012, Cuiabá.

Crossland, M.D. et al. (2014). "Smartphone, tablet computer and e-reader use by people with vision impairment”. Ophthalmic and Physiological Optics. 34, 5 (2014), 552557.

Guerreiro, T. et al. (2009). "NavTap: A Long Term Study with Excluded Blind Users". In Proceedings of the 11th International ACM SIGACCESS Conference on Computers and Accessibility (New York, NY, USA, 2009), 99-106.

Kane, S.K. et al. (2011). Usable Gestures for Blind People: Understanding Preference and Performance. In Proceedings of the SIGCHI Conference on Human Factors in Computing Systems (New York, NY, USA, 2011), 413-422. 
Issa. et. al. (2009). "Accessibility for the blind: an automated audio / tactile description of pictures in digital documents" In International Conference on Advances in Computational Tools for Engineering Applications, 2009. (ACTEA '09). Zouk Mosbeh, Lebanon, 15-17 July.

Jayant, C. et.al. (2010). "VBraille: Haptic Braille Perception using a Touch-screen and Vibration on Mobile Phones", In Proceedings of the 12th international ACM SIGACCESS conference on Computers and Accessibility (․ASSETS '10). New York, NY, USA, 295-296.

Jr, J. and Hayden, D. (2010). "The Note-Taker: An Assistive Technology That Allows Students Who Are Legally Blind to Take Notes in the Classroom". IEEE Computer Society Conference on Computer Vision and Pattern Recognition Workshops (CVPRW). Proceedings do IEEE 13-18 June,2010.

Kane, S.K. et al. (2011). Usable Gestures for Blind People: Understanding Preference and Performance. Proceedings of the SIGCHI Conference on Human Factors in Computing Systems (New York, NY, USA, 2011), 413-422.

Kitchenham, B. (2004). Procedures for Performing Systematic Reviews. Joint Technical Report,TR / SE-0401 and NICTA 0400011T.1, Keele University.

Leporini, B. et al. (2012). Interacting with Mobile Devices via VoiceOver: Usability and Accessibility Issues. In Proceedings of the 24th Australian Computer-Human Interaction Conference (New York, NY, USA, 2012), 339-348.

Mehigan, T. (2009). "Harnessing Accelerometer Technology for Inclusive Mobile Learning". In Proceedings of the 11th International Conference on HumanComputer Interaction with Mobile Devices and Services (MobileHCI '09). New York, NY,USA. Article 100, 2 pages.

Milne et.al. (2014). "BraillePlay: Educational Smartphone Gamesfor Blind Children", In Proceedings of the 16th international ACM SIGACCESS conference on Computers \& Accessibility (ASSETS '14). Rochester, New York, USA, October,137144.

Raisamo et.al. (2007). "Design and evaluation of a tactile memory game for visually impaired children”. Interacting with Computers, vol. 19,pp. 196-205.

Southern. et.al. (2012) “An Evaluation of BrailleTouch: Mobile Touchscreen Text Entry for the Visually Impaired", In Proceedings of the 14th international conference on Human-computer interaction with mobile devices and services (MobileHCI '12).New York,NY,USA,317-326.

Zapirain,et. al. (2010). “Accessible schematics content descriptors using image processing techniques for blind students Learning", In Proceedings of the 5th International Symposium on I/V Communications and Mobile Network (ISVC), pp.1-4. 